\title{
Erratum to: Thermodynamic Description of the Ternary Sb-Sn-Zn System
}

\author{
WOJCIECH GIERLOTKA ${ }^{1,2}$ \\ 1.-National Dong Hwa University, Hualien, Taiwan, ROC. 2.-e-mail: wojtek@mail.ndhu.edu.tw
}

\section{Erratum to: Journal of ELECTRONIC \\ MATERIALS, Vol. 45, No. 4, 2016 \\ DOI: 10.1007/s11664-015-4276-5}

In the original article there was an error in the author's affiliation. It is corrected as shown in this erratum. 